\title{
Variación en la composición de comunidades de aves en la Reserva de la Biosfera Montes Azules y áreas adyacentes, Chiapas, México
}

\author{
Jorge E. Ramírez-Albores ${ }^{1,2,3}$ \\ Biota Neotropica v6 (n2) - http://www.biotaneotropica.org.br/v6n2/pt/abstract?article+bn03106022006 \\ Recebido em 09/09/2005 \\ Versão Reformulada recebida em 25/05/2006 \\ Publicado em 28/06/2006 \\ ${ }^{1}$ El Colegio de La Frontera Sur (ECOSUR), San Cristóbal de las Casas, Chiapas, México. \\ ${ }^{2}$ BALAM-HA S. A de C. V. México, Distrito Federal. México. \\ ${ }^{3}$ Dirección postal: Manuel Bonilla \# 357 Manzana 44 Col. Santa Martha Acatitla C. P. 09510, Delegación Iztapalapa. \\ México, Distrito Federal. México. (jorgeramirez22@hotmail.com)
}

\begin{abstract}
Ramírez-Albores, J.E. Variability in bird communities' composition in the Biosphere Reserve Montes Azules and adjacent areas, Chiapas, Mexico. Biota Neotrop. May/Aug 2006 vol. 6 no. 2, http://www.biotaneotropica.org.br/v6n2/pt/ abstract?article+bn03106022006. ISSN 1676-0603
\end{abstract}

In order to compare the bird communities’ composition and their possible relationships, I sampled monthly from January to December 2002 in the Biosphere Reserve Montes Azules (REBIMA) and adjacent areas, Chiapas, Mexico. Data were obtained during 48 days of fieldwork in an area of 60 ha by site. I recorded 133 species, which represent $36 \%$ of the total number of species reported for the REBIMA. Highest species richness was found in the site 1 (well preserved forest with 113 spp). High similarity between the sites 1 and 4 were found (this last with $50 \%$ original vegetation, IS $=0.89$ ). Approximately $80 \%$ of the species were residents; and insectivores were the best represented trophic guild (47 spp). All feeding guilds were represented in all sites showing differences in species richness and abundance. Variation in the richness and composition of bird communities among different sizes was due to the fact that some species may demonstrate a different response to the degree of forest fragmentation and habitat transformation. However, in spite of limited sampling efforts, the bird richness suggests that secondary habitats play an important role in the persistence of species in tropical ecosystems.

Key words: richness, similarity, tropical forest, Selva Lacandona.

\section{Resumen}

Ramírez-Albores, J.E. Variación en la composición de comunidades de aves en la Reserva de la Biosfera Montes Azules y áreas adyacentes, Chiapas, México. Biota Neotrop. May/Aug 2006 vol. 6 no. 2, http://www.biotaneotropica.org.br/ v6n2/pt/abstract?article+bn03106022006. ISSN 1676-0603

Con el fin de comparar la composición de las comunidades de aves y su posible interrelación, se realizaron muestreos mensuales entre Enero-Diciembre de 2002 en la Reserva de la Biosfera Montes Azules (REBIMA) y áreas adyacentes, Chiapas, México. Los datos fueron generados durante 48 días de observaciones de campo en un área de 60 ha por sitio. Se registraron 133 especies que representan el 36\% de las especies de aves reportadas para la REBIMA. La mayor riqueza de especies de aves fue registrada en el sitio 1 (sitio con selva intacta ubicado en la REBIMA, 113 spp). El sitio 1 mostró una mayor similitud con el sitio 4 (sitio con el 50\% de vegetación original, IS = 0,89). El 80\% de las especies fueron residentes y el gremio mejor representado fue el de las especies insectívoras (47 spp). Los sitios presentaron los mismos gremios alimenticios con diferencias en su riqueza y abundancia de especies. Algunas especies de aves parecen responder de forma diferente al grado de fragmentación y transformación del hábitat, por lo que se presenta una variación en la riqueza y en la composición de las comunidades de aves en los diferentes sitios. Sin embargo, a pesar del limitado esfuerzo de muestreo, la riqueza avifaunística registrada en este estudio demostró que los hábitats secundarios juegan un papel importante en la persistencia de las especies de aves en ecosistemas tropicales.

Palabras-clave: riqueza, similitud, selva tropical, Selva Lacandona. 


\section{Introducción}

La distribución y abundancia de las aves son el resultado de la influencia tanto de factores históricos como ecológicos (Hutto 1985). Entre los diversos factores ecológicos más importantes se encuentran la estructura del hábitat (usualmente medida a través de valores que describen a la estructura vegetal) y la disponibilidad del alimento (la abundancia de presas potenciales dentro del microhábitat utilizado por un ave) (Wolda 1990). Los aspectos de la vegetación varían de acuerdo con la escala espacial (a un nivel de paisaje y de hábitat) y la escala temporal, los que a su vez determinan la disponibilidad del recurso alimenticio (Wiens \& Rotenberry 1981).

Las especies de aves neotropicales, cuando se desplazan en busca de mejores condiciones alimentarías, climáticas o reproductivas, producen cambios en la composición de las comunidades (Verea et al. 2000). Este fenómeno está bien estudiado en especies migratorias que cubren grandes distancias desde las zonas templadas hacia el trópico (Greenberg 1986, Rappole 1995). Aunque existen datos sobre el movimiento horizontal de muchas especies locales en busca de mejores condiciones alimentarías entre ambientes (Karr 1977, Karr et al. 1982, Poulin et al. 1994) así como sobre ciertos movimientos verticales dentro de los estratos de un ambiente particular (Verea \& Solórzano 1998) los estudios de estas variaciones son escasos.

En México, la vegetación de selva húmeda tropical ha sido reducida a menos del $10 \%$ de su cobertura original (Ramos 1985). Existen actualmente sólo remanentes de selvas; uno de ellos es la Reserva de la Biosfera de Montes Azules (REBIMA) ubicada en la región conocida como La Selva Lacandona, en el estado de Chiapas (sureste de México) la cual es identificada como una de las principales zonas prioritarias para la conservación. Esta región es uno de los relictos más importantes del país en cuanto a diversidad biológica y cultural. Sin embargo, factores como la deforestación, crecimiento de la frontera agrícola, creación de caminos y accesos a la zona, crecimiento desmedido de la población humana y uso inmoderado de recursos, han tenido un efecto en las poblaciones de especies silvestres, provocando una alteración de su hábitat natural, lo cual ocasiona un cambio en la distribución y abundancia de especies (INE-Semarnap 2000).

Diversos estudios revelan la importancia de esta región por su gran riqueza y composición avifaunística (Nocedal 1981, Rangel-Salazar 1990, González-García 1993, Rangel-Salazar et al. 1993, Warkentin et al. 1995, Greenberg 1996, Puebla-Olivares et al. 2002) pero ninguno ha evaluado las relaciones en su composición entre sus ambientes. Para contribuir al entendimiento de las variaciones ambientales y estaciónales, además de la variación en la riqueza y la composición de las comunidades de aves en sitios con diferentes grados de perturbación de selva tropical, se compara la estructura avifaunística en la Reserva de la
Biosfera de Montes Azules y áreas adyacentes, esto con el fin de aportar datos que favorezcan el manejo y conservación de las comunidades faunísticas que habitan en estas áreas.

\section{Material y Métodos}

\section{1. Área de estudio}

El área de estudio se ubica en la Reserva de la Biosfera

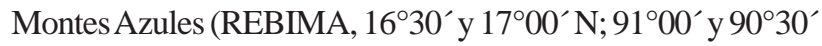
W; superficie 331200 ha) y en áreas adyacentes (Figura 1, Tabla 1). Los sitios de muestreo se ubicaron en la comunidad de Playón de la Gloria (1609 N, 9053’ W, a una altitud de 200 msnm) en el municipio de Ocosingo (Figura 1, Tabla 1). La REBIMA se encuentra en la región conocida como Selva Lacandona cuenta con un relieve heterogéneo, incluyendo lomeríos, planicies inundables asociadas a los ríos principales (Lacantún, Lacanjá) con una altitud entre 150 y 200 msnm y serranías de hasta 1200 msnm con numerosas cañadas (Castillo-Campos \& Narave 1992, INE-Semarnap 2000). El clima predominante en el área es cálido húmedo. La precipitación media anual es superior a $2500 \mathrm{~mm}$, con una estación seca corta de Febrero a Mayo y la temperatura media anual es generalmente superior a los $24^{\circ} \mathrm{C}$. La mayor parte de la vegetación de esta zona está constituida por selva alta perennifolia (Miranda \& Hernández 1963) y remanentes de selva tropical mediana. La vegetación secundaria más frecuente corresponde a los acahuales, que actualmente cubren considerables extensiones en todas las zonas perturbadas de la selva, encontrándose en diversas etapas de regeneración. Algunas especies vegetales comunes en la selva de la región son Spondias mombin (Anacardiaceae), Ceiba pentandra (Bombaceae), Terminalia amazonia (Leguminosae), Dialium guianense (Leguminosae), Swietenia macrophylla (Leguminosae), Brosimum alicastrum (Moraceae), Ficus insipida (Moraceae), Bactris balanoidea (Palmae), Licania platypus (Rosaceae) y Pouteria sapota (Sapotaceae) (INE-Semarnap 2000).

\section{Colecta de datos}

Se realizaron salidas de campo mensuales de Enero a Diciembre de 2002 con un total de 48 días de trabajo de campo. Se llevaron a cabo recorridos de observación en transectos (Emlen 1971) de 2 a 3 km de longitud en un área de muestreo de 60 ha por sitio. Se efectuaron 48 transectos por sitio, para un total de 192 transectos. La distancia aproximada entre el sitio de muestreo de la REBIMA con los otros sitios varió de 3 a $5 \mathrm{~km}$. Las aves se identificaron por observación directa con la ayuda de binoculares (10 x 50) y registros auditivos. Se utilizaron las guías de aves de Peterson \& Chalif (1994) y Howell \& Webb (1995) para la identificación de las especies. La nomenclatura taxonómica se apega a la propuesta por la American Ornithologists' Union (1998, 2000) y Banks et al. (2002, 2003, 2004, 2005). 


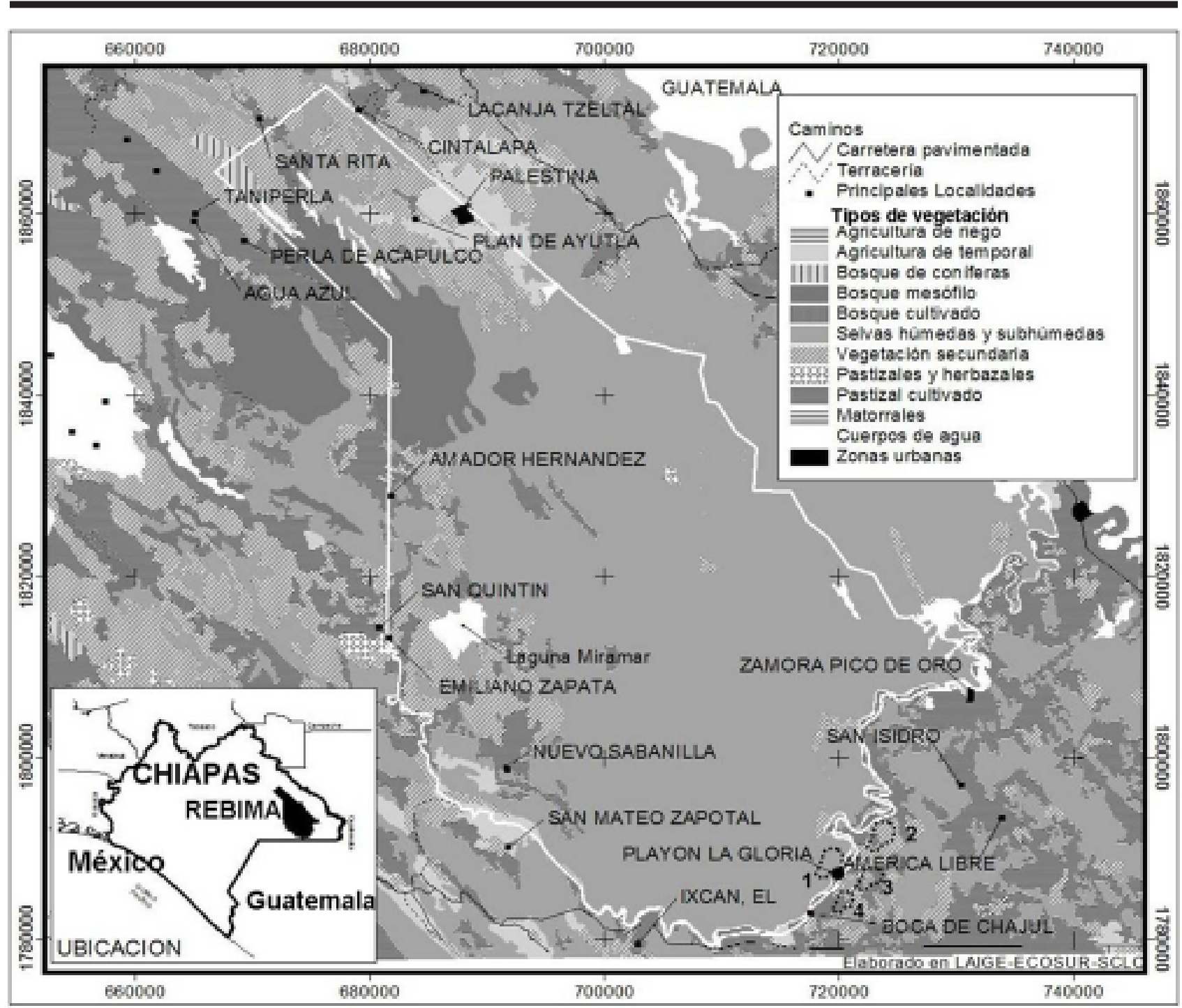

Figura 1- Ubicación geográfica de la Reserva de la Biosfera Montes Azules y áreas adyacentes, Chiapas, México. Sitios de muestreo: Sitio 1 (sitio dentro de la REBIMA, presenta el 100\% de vegetación original), Sitio 2. (sitio adyacente a la reserva, presenta manchones de selva del 10 al 20\% de vegetación original asociado con pastizales), Sitio 3 (sitio adyacente a la reserva, presenta manchones de selva del 20 al $30 \%$ de vegetación original asociado con plantaciones de cacao, pastizales y vegetación secundaria), Sitio 4 ( sitio adyacente a la reserva, presenta manchones de selva con el 50\% de vegetación original asociado con vegetación secundaria y pastizales).

Figure 1- Geographic ubication of the Biosphere Reserve Montes Azules and adjacent areas, Chiapas, Mexico. Study sites: Site 1 (site in the REBIMA, with $100 \%$ of original vegetation), Site 2 (adjacent site to the reserve, with forest remnants of 10 to $20 \%$ of original vegetation associated with pastures), Site 3 (adjacent site to the reserve, with forest remnants of 20 to $30 \%$ of original vegetation associated with cacao plantations, pastures and secondary vegetation), Site 4 (adjacent site to the reserve, with forest remnants of 50\% of original vegetation associated with secondary vegetation and pastures). 
Tabla 1. Descripción de los sitios de estudio en la Reserva de la Biosfera Montes Azules y áreas adyacentes, Chiapas, México.

Table 1. Description of the study sites in the Biosphere Reserve Montes Azules and adjacent areas, Chiapas, Mexico.

\begin{tabular}{|c|c|c|c|c|}
\hline $\begin{array}{l}\text { Sitios de } \\
\text { estudio }\end{array}$ & Ubicación & Altitud & Tipos de vegetación & $\begin{array}{c}\% \text { de } \\
\text { vegetación } \\
\text { original }\end{array}$ \\
\hline Sitio 1 & $\begin{array}{l}\text { sitio dentro de la REBIMA, ubicado a } \\
1 \text { kilómetro al norte de Playón de la } \\
\text { Gloria }\end{array}$ & $50 \mathrm{~m}$ & $\begin{array}{l}\text { Selva alta perennifolia y } \\
\text { selva mediana perennifolia }\end{array}$ & $100 \%$ \\
\hline Sitio 2 & $\begin{array}{l}\text { sitio adyacente a la reserva, ubicado a } \\
3 \text { kilómetros al este de Playón de la } \\
\text { Gloria }\end{array}$ & $200 \mathrm{~m}$ & $\begin{array}{l}\text { manchones de selva } \\
\text { mediana perennifolia } \\
\text { asociado con pastizales }\end{array}$ & $10-20 \%$ \\
\hline Sitio 3 & $\begin{array}{l}\text { sitio adyacente a la reserva, ubicado a } \\
1.5 \text { kilómetros al sur de Playón de la } \\
\text { Gloria }\end{array}$ & $110 \mathrm{~m}$ & $\begin{array}{l}\text { manchones de selva } \\
\text { mediana perennifolia } \\
\text { asociado con plantaciones } \\
\text { de cacao, pastizales y } \\
\text { vegetación secundaria }\end{array}$ & $20-30 \%$ \\
\hline Sitio 4 & $\begin{array}{l}\text { sitio adyacente a la reserva, ubicado a } \\
2 \text { kilómetros al oeste de Playón de la } \\
\text { Gloria }\end{array}$ & $200 \mathrm{~m}$ & $\begin{array}{l}\text { manchones de selva } \\
\text { mediana perennifolia } \\
\text { asociado con pastizales y } \\
\text { vegetación secundaria }\end{array}$ & $50 \%$ \\
\hline
\end{tabular}


Para evaluar el esfuerzo de muestreo, se construyeron curvas de acumulación de especies por sitio de muestreo y para el área de estudio mediante el modelo de estimación de riqueza de Clench [Función de Clench $S(t)=$ at/ $(1+\mathrm{bt})$ ] donde $S(t)$ es el número esperado de especies después de $t$ conteo por puntos, $a$ es el incremento del valor de la lista de especies al comienzo de la colecta con unidades de especies $x$ conteo por puntos ${ }^{-1}, b$ es el incremento del valor con unidades de conteo por puntos ${ }^{-1}$. La asíntota estimada o la predicción de la riqueza de especies fueron calculadas como $a / b$ (Soberón \& Llorente 1993, Colwell \& Coddington 1994). Para la construcción de las curvas de acumulación de especies esperadas se utilizó el programa estadístico STATISTICA ${ }^{\circledR} 6$.

La lista de especies obtenida fue comparada con inventarios de zonas relativamente cercanas para confirmar la presencia de las especies registradas en este estudio (Nocedal 1981, Rangel-Salazar 1990, González-García 1993, Rangel-Salazar et al. 1993, Warkentin et al. 1995, Greenberg 1996, Puebla-Olivares et al. 2002). Adicionalmente, se usaron los criterios tales como la falta de especies en determinadas familias o géneros, así como el registrar menos de 35 especies y 21 familias, entre otros para sugerir si el inventario esta aproximadamente completo (Gómez de Silva \& Medellín 2001).

Con los datos obtenidos en los sitios de muestreo, se calculó su similitud usando el índice de Sorensen (IS = 2S/ $\mathrm{N}_{1}+\mathrm{N}_{2}$, donde $\mathrm{S}$ es el número de especies compartidas entre el sitio $\mathrm{A}$ y al sitio $\mathrm{B} ; \mathrm{N}_{1}$ es el número total de especies del sitio A y $\mathrm{N}_{2}$ es el número de especies del sitio B). Este índice tiene un intervalo que va desde 0 , cuando no hay especies en común entre las muestras, hasta 1 cuando ambas muestras son idénticas en cuanto a su composición de especies (Ravinovich 1981).

La estacionalidad se determinó de acuerdo a Howell \& Webb (1995) como: visitante de invierno, residente de verano, transitoria, vagabunda, residente y ocasional. Los hábitos alimenticios de las especies se determinaron por las observaciones en campo que fueron complementadas con información bibliográfica. Las categorías asignadas representaron la dieta y subdieta más común de las especies como: insectívoras, frugívoras, nectarívoras, carnívoras, granívoras, omnívoras, carnívora-insectívora, carnívorasinsectívoras-frugívoras, granívoras-frugívoras, granívorasinsectívoras, granívoras-insectívoras-frugívoras, insectívoras-frugívoras e insectívoras-nectarívoras.

\section{Resultados}

Un total de 133 especies fueron registradas en los sitios de estudio (Apéndice 1). Las familias mejor representadas fueron los Accipitridae, Columbidae, Tyrannidae, Parulidae e Icteridae (Apéndice 1).

En el sitio 1 se encontró la mayor riqueza de especies (113), seguido por el sitio 4 con 88 especies, el sitio 2 con 65 y el sitio 3 con 64 (Figuras 2, 3). De acuerdo con el estimador de riqueza de especies, en el sitio 1 podrían registrarse 125 especies, 80 en el sitio 2, 94 en el sitio 3 y 103 en el sitio 4 (Figuras 2, 3). La riqueza estimada para toda el área de estudio es de 146 especies (Figuras 2, 3).

El promedio de riqueza mensual por sitios fue mayor en el sitio 1 (48 especies) y el más bajo fue en el sitio 3 (40 especies) (Figuras 2, 3). En el mes de Octubre se registro la mayor riqueza y abundancia de especies en los sitios de estudio (Figuras 2 a, b). La menor riqueza y abundancia de especies en los sitios de estudio fue en el mes de Mayo, que es considerado como el más seco de ese año (Figuras 2, 3).

Con relación a la similitud, el sitio 1 compartió un mayor número de especies con el sitio 4 (IS $=0,89$ ), seguido por el sitio 3 (IS = 0,77), mientras que el sitio 2 compartió un menor número de especies (IS =0,46). Se encontraron 20 especies compartidas en los cuatro sitios de muestreo, 13 especies fueron exclusivas del sitio 1 , dos especies en el sitio 2, una especie en el sitio 3 y una especie en el sitio 4 (Apéndice 1).

Del total de especies registradas, 106 son residentes (80\%) y el resto son migratorias (20\%; Tabla 2, Apéndice 1). La mayor riqueza de especies residentes se registro en el sitio 3 (87\%) y en el sitio 4 (87\%); mientras que en el sitio 1 fue del 74\% y en el sitio 2 de 65\% (Tabla 2, Apéndice 1). En cuanto a las especies migratorias, la mayor riqueza se registro en el sitio 2 (22\%), y en el sitio 3 se registró el menor número de especies (7, 11\%) (Tabla 2). Las especies Progne chalybea, Polioptila caerulea y Piranga rubra estuvieron representados en todos los sitios, con una mayor abundancia en el sitio 3.

En cuanto a los hábitos alimenticios, las especies insectívoras estuvieron mejor representadas (47 spp.), seguidas por las especies carnívoras (23 spp.) y las especies insectívoras-frugívoras (21 spp.; Tabla 2, Apéndice 1). En el sitio 1, las especies insectívoras presentaron la mayor riqueza de especies (39 spp.), mientras que en el sitio 2 sólo fue de 15 especies (Tabla 2, Apéndice 1). Al analizar la similitud en la composición de especies con base a los hábitos alimenticios, el sitio 3 compartió un mayor número de especies insectívoras con el sitio 1 (IS = 0,91); a diferencia de los sitios 2 y 4 que compartieron un menor número de especies (IS = 0,37 y IS =0,48, respectivamente). Las especies insectívoras tales como Chaetura vauxi, Dryocopus lineatus, Lepidocolaptes souleyetii, Tyrannus melancholicus y Thryothorus maculipectus estuvieron representados en todos los sitios, con una mayor abundancia en el sitio 1 .

Por otra parte, el sitio 1 compartió un mayor número de especies carnívoras con el sitio 4 (IS $=0,96$ ) y el sitio 2 (IS =0,72), $\mathrm{y}$ el sitio 3 con un menor número de especies compartidas (IS = 0,47). Para los granívoros-frugívoros, el sitio 1 compartió un mayor número de especies con el sitio 4 (IS $=0,96$ ), seguido por el sitio 3 (IS $=0,84$ ) y el sitio 2 (IS 


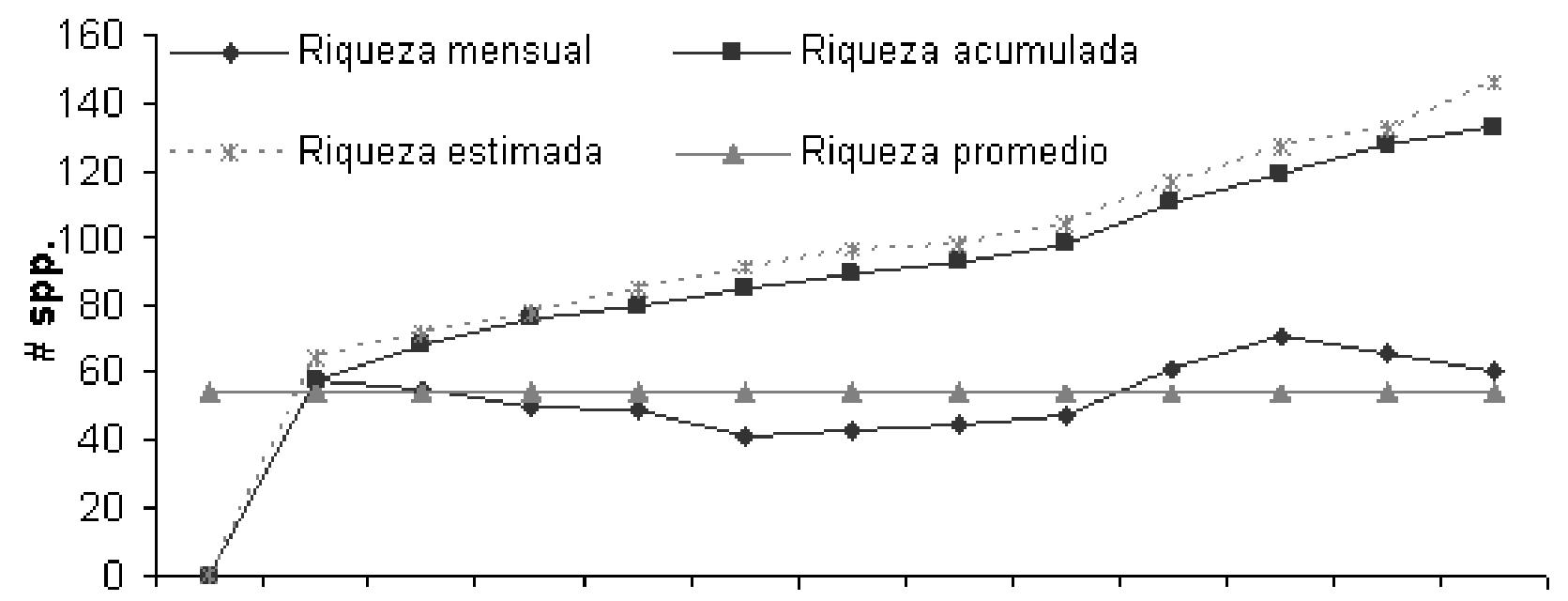

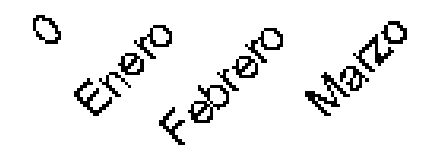

Área de estudio

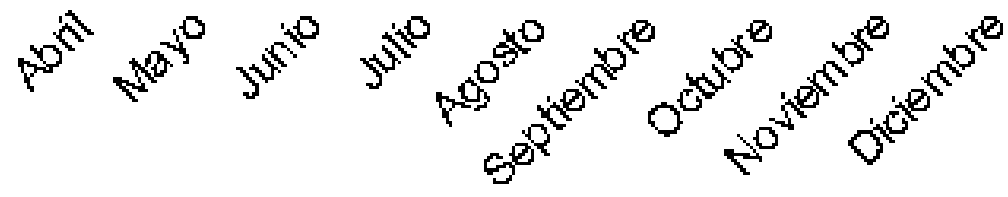

esfuerzo de muestreo (mes)

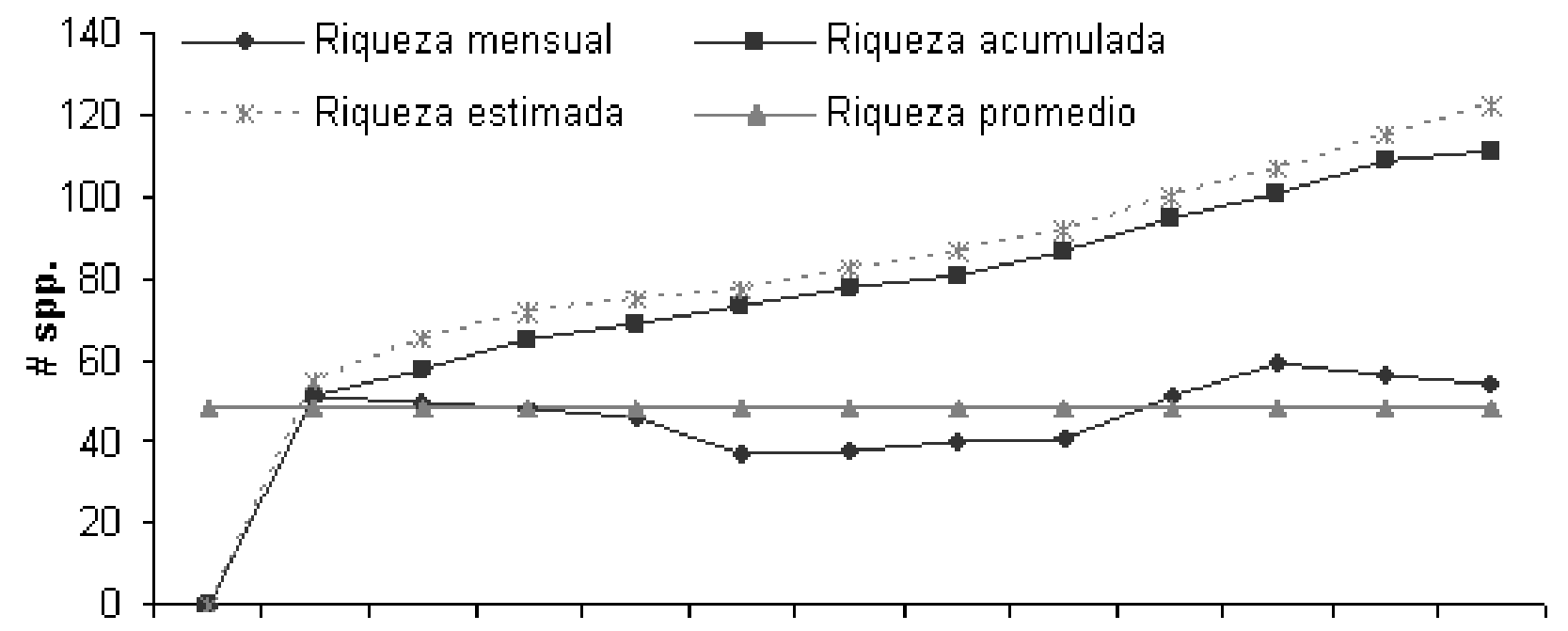

Sitio 1

0

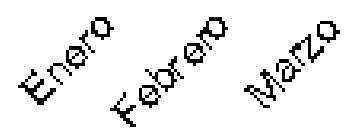

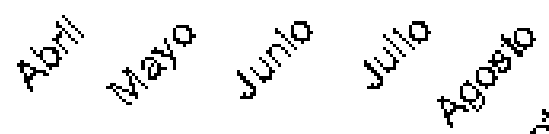

$0^{\circ}$

esfuerzo de muestreo (imes)

Figura 2- Acumulación de especies observadas y estimadas en el área de estudio y en el sitio de estudio en la Reserva de la Biosfera Montes Azules, Chiapas, México.

Figure 2- Cumulative number of species observed and estimated in the study area and study site in the Biosphere Reserve Montes Azules, Chiapas, Mexico. 


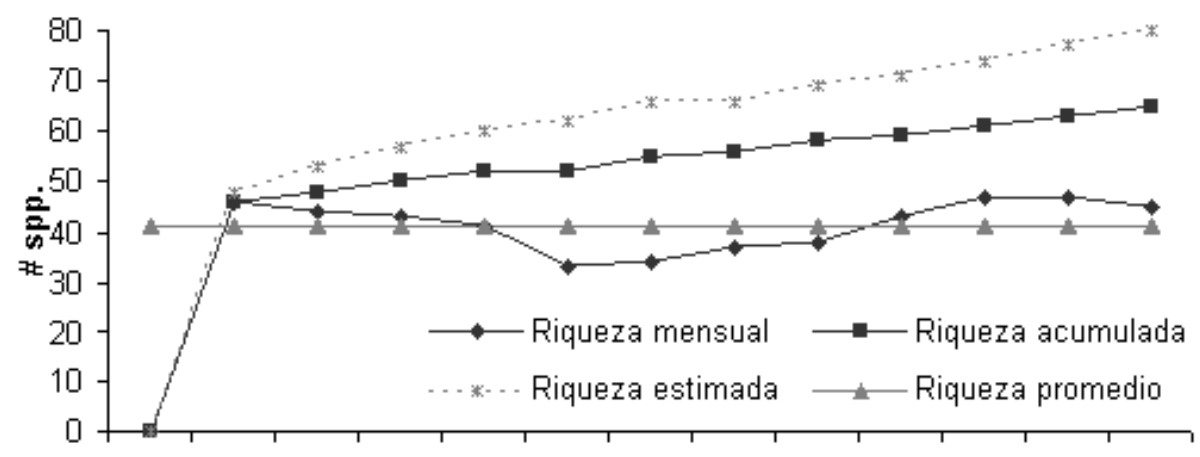

$\bigcirc$

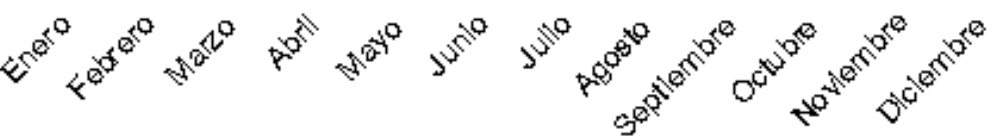

Sitio 2

esfuerzo de muestreo (mes)

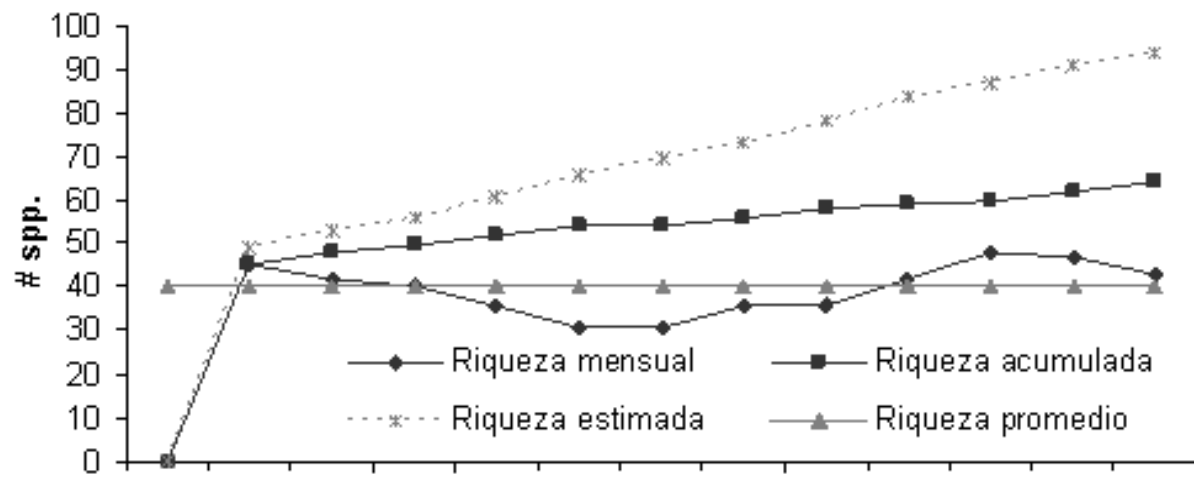

○

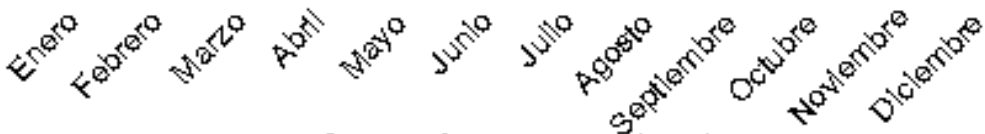

Sitio 3

esfuerzo de muestreo (mes)

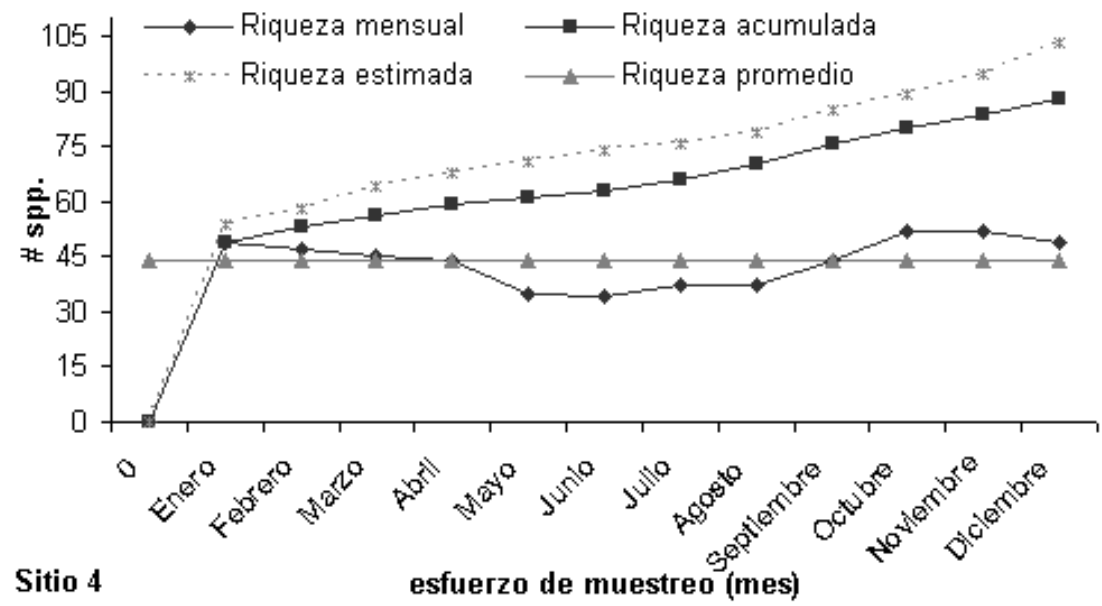

Figura 3- Acumulación de especies observadas y estimadas en los sitios de estudio adyacentes a la Reserva de la Biosfera Montes Azules, Chiapas, México.

Figure 3- Cumulative number of species observed and estimated in the adjacent study sites at Biosphere Reserve Montes Azules, Chiapas, Mexico.

http://www.biotaneotropica.org.br 
Tabla 2. Número de especies en los sitios de estudio en la Reserva de la Biosfera Montes Azules y áreas adyacentes, Chiapas, México, en función del estatus y hábitos alimenticios.

Table 2. Number of species in the study sites in the Biosphere Reserve Montes Azules and adjacent areas, Chiapas, Mexico, according to the status and feeding habits.

\begin{tabular}{|c|c|c|c|c|c|}
\hline & Total & $\begin{array}{c}\text { Sitio } \\
1\end{array}$ & $\begin{array}{c}\text { Sitio } \\
2\end{array}$ & $\begin{array}{c}\text { Sitio } \\
\mathbf{3}\end{array}$ & $\begin{array}{c}\text { Sitio } \\
4\end{array}$ \\
\hline \multicolumn{6}{|l|}{ Estatus } \\
\hline Residentes & 106 & 89 & 42 & 56 & 77 \\
\hline Visitantes de invierno & 22 & 20 & 15 & 6 & 11 \\
\hline Transitorias & 3 & 2 & 2 & 1 & \\
\hline Residentes de verano & 2 & 2 & 1 & 2 & 1 \\
\hline \multicolumn{6}{|l|}{ Hábitos alimenticios } \\
\hline Carnívoras & 22 & 13 & 12 & 8 & 12 \\
\hline Carnívoras-insectívoras & 4 & 4 & 3 & & 1 \\
\hline Carnívoras-insectívoras-frugívoras & 1 & 1 & & 1 & 1 \\
\hline Granívoras & 6 & 1 & 6 & 5 & 2 \\
\hline Granívoras-frugívoras & 16 & 15 & 4 & 11 & 16 \\
\hline Granívoras-insectívoras & 1 & & 1 & 1 & \\
\hline Granívoras-insectívoras-frugívoras & 4 & 2 & 4 & 3 & 2 \\
\hline Insectívoras & 46 & 39 & 15 & 20 & 27 \\
\hline Insectívoras-frugívoras & 21 & 19 & 7 & 10 & 17 \\
\hline Insectívoras-nectarívoras & 1 & 1 & & & 1 \\
\hline Nectarívoras & 5 & 5 & 3 & 2 & 5 \\
\hline Omnívoras & 6 & 4 & 2 & 2 & 4 \\
\hline
\end{tabular}

http://www.biotaneotropica.org.br 
= 0,42). Para los omnívoros, el sitio 1 compartió un mayor número de especies con el sitio 4 (IS $=0,85$ ), seguido por el sitio 2 y 3 con una similitud de 0,80 cada uno. Para los insectívoros-frugívoros, el sitio 1 compartió un mayor número de especies con el sitio 4 (IS $=0,85$ ), seguido por el sitio 3 (IS $=0,50$ ) y el sitio 2 (IS = 0,48). Para los nectarívoros, el sitio 1 compartió todas las especies con el sitio 4 (IS =1,00), seguido por el sitio 2 (IS $=0,75$ ) y el sitio 3 (IS $=0,57$ ). De la misma forma para los granívoros-insectívoros-frugívoros, el sitio 1 compartió todas las especies con el sitio 4 (IS = 1,00), seguida por el sitio $3($ IS $=0,80$ ) y el sitio 2 (IS $=0,66$ ).

\section{Discusión}

En el presente estudio se registraron un total de 133 especies, lo que corresponde al 36\% de las especies de aves reportadas para la Reserva de la Biosfera de Montes Azules (Nocedal 1981, Rangel-Salazar 1990, González-García 1993).

Los resultados aquí presentados sugieren que la riqueza y abundancia de especies varía en los sitios de estudio. El sitio 1(selva madura) parece ser atractivo para un mayor número de especies de aves, ya que tanto la riqueza como la abundancia fueron más altas en este; lo que concuerda con algunos trabajos realizados en condiciones similares (Blake \& Loiselle 1991, Estrada et al. 1997, Blake \& Loiselle 2001, Bojorges \& López-Mata 2001). En este sentido se ha señalado que la estratificación tanto horizontal como vertical de la vegetación podría promover la coexistencia de un mayor número de especies de aves (Levey 1988, Blake \& Loiselle 1991), ya que estas podrían utilizar la cobertura protectora y recursos alimenticios disponibles (Terborgh \& Weske 1969, McIntyre 1995). De acuerdo a Blake \& Loiselle (2001), en ambientes que generalmente presentan altas tasas de producción de flores, frutos y follaje, pueden albergar mayor riqueza y abundancia de especies.

En los sitios 2, 3 y 4 (ambientes sucesionales), la variación de la riqueza y abundancia de especies de aves obedece a cambios en la vegetación y a movimientos temporales de las aves debido a la disponibilidad del alimento. En este sentido, la variación de la riqueza en cada uno de los sitios de estudio podría ser explicada en términos tróficos. Por ejemplo, los tiránidos aunque fueron más ricos en el sitio 1, fueron más abundantes en los sitios adyacentes a la REBIMA, esto debido a que están representados por especies que prefieren hábitat más abiertos (Anjos et al. 1997), donde tienen una mayor visibilidad y libertad de movimientos para conseguir alimento (Orinas 1969).

Por otra parte, aunque se ha considerado que ambientes maduros (e.g., selvas) son muy diversos (Blake \& Loiselle 2001), varios investigadores han señalado que tanto la riqueza como la abundancia y diversidad de especies es similar en etapas de sucesión tempranas y maduras (Andrade \& Rubio 1994, Petit et al. 1995, Smith et al. 2001). Por ejemplo, los resultados aquí presentados varían de acuerdo a lo reportado por Robinson \& Terborgh (1997) y Blake \& Loiselle (2001) en selvas altas de Costa Rica, en donde selvas en regeneración presentaron mayor riqueza y abundancia de especies de aves que la selva madura. Estos patrones no se observaron en este estudio, lo que pudiera ser consecuencia de la extensión del área, de la variación de la abundancia de cada especie y de su preferencia de área.

Los sitios de muestreo adyacentes a la REBIMA mostraron semejanzas en el número de especies, por ejemplo en los sitios 3 y 4, estuvieron presentes el 77\% y 89\% del total de las especies registradas en el sitio 1 , respectivamente. Por otra parte, el sitio 2 mostró una baja similitud con el sitio 1 , solo el $46 \%$ del total de las especies estuvo presente en este sitio. En el sitio 1 estuvieron presentes todas las familias reportadas para la REBIMA, mientras que en los sitios adyacentes a la REBIMA no estuvieron representadas todas las familias. Karr (1990) y Staicer (1992) sugieren que un mosaico de ambientes puede ser utilizado para diferentes propósitos y permite a las aves cambiar su distribución como respuesta a las condiciones de estos. Sin embargo, la similitud de los sitios de estudio indica la existencia de un intercambio relativamente bajo de especies, así como de una aparente baja conectividad entre estas áreas (sensu Wiens 1997), lo anterior sugiere que tanto la configuración del ambiente (e.g., paisaje, hábitat y microhábitat) como la cantidad disponible de estos no serían igualmente importantes en la distribución de las aves (Karr 1990) y podría conferir cambios en la composición de la comunidad de aves (Blake \& Loiselle 2001). Sin embargo, en ambientes maduros (e.g., selvas) la riqueza y abundancia de las especies tienen variaciones que difieren de acuerdo con el tipo de sistema, al grado de variabilidad ambiental del mismo, y a la escala espacio-temporal del análisis (Lord \& Norton 1990, López de Casenave \& Marone 1996).

Aunque en algunos ambientes tropicales es bien conocido que las especies de aves migratorias son capaces de producir cambios en la composición de las comunidades de aves tropicales (Karr et al 1982), en este estudio las especies migratorias (20\%) jugaron un papel menor en los cambios observados en la comunidad de aves. Esto concuerda con los resultados de otros estudios en ambientes tropicales, como en "La Selva” en Costa Rica donde el 21\% son migratorias (Stiles 1983, Blake et al. 1990) o en Barro Colorado, en Panamá, donde sólo se registró el 22\% de aves migratorias (Willis 1980, Karr 1990, Robinson et al. 2000). En México, Nocedal (1981) registró que el 20\% eran migratorias para la región de Lacanjá-Chansayab. González-García (1993) reportó que el 21\% del total son migratorias para la Reserva de la Biosfera de Montes Azules. Puebla-Olivares et al. (2002) reportan que el $26 \%$ de las especies son migratorias para la región de Yaxchilán en la parte este de la Selva Lacandona. En la región de Los Tuxtlas en Veracruz, se ha reportado el 15\% de migratorias (Winker et al. 1992, Estrada et al. 1997, 2000). Bojorges y López-Mata (2001, 2005) reportan el 34\% de migratorias en el centro de Veracruz. 
Posiblemente existe una tendencia, entre más al sur y al oeste se encuentre un sitio (en Norte y Centro América) la proporción de especies migratorias disminuye. Por ejemplo, en Chamela-Cuixmala (al oeste) el 38\% de la avifauna es migratoria (Arizmendi et al. 1990) y en Los Tuxtlas (al sur) el 15\% de la avifauna es migratoria (Winker et al. 1992, Estrada et al. 1997, 2000). La tendencia hacia el sur podría estar relacionada con la lejanía de los sitios de anidación de las especies migratorias, y hacia el Este y Oeste con la riqueza avifaunística de cada zona en Estados Unidos y Canadá(OrtizPulido 1994) y debido a los calendarios de migración, donde una gran proporción de especies de aves migran entre la parte este de los Estados Unidos y las zonas tropicales de Centroamérica a lo largo del Golfo de México durante el verano (Abril-Mayo) y otoño (Septiembre-Octubre) (Buskirk 1980, Hespenheide 1980, Mills \& Rogers 1990).

La mayor riqueza y abundancia en los sitios de muestreo fue durante la época de lluvias, principalmente en los meses de Octubre y Noviembre (Cuadro 2). El promedio de especies por muestra mensual resultó superior en el sitio 1 (sitio de muestreo dentro de la REBIMA), probablemente por que constituye un área importante para muchas especies de aves que son atraídas por la presencia de hábitat primarios y sucesionales, las condiciones climáticas, el suministro continuo de agua, alimento y refugio (González-García 1993). A diferencia de esto, en los sitios 2 y 3 los promedios de riqueza mensual fueron bajos (Figura 2) donde el grado de cobertura de selva y los cambios en la vegetación (i.e., pastizales y cultivos) pudo haber influido en la riqueza de especies de aves en estos sitios. Se puede establecer que temporalmente cada uno de los sitios manifiesta su propia riqueza y abundancia de especies. Loiselle \& Blake (1992) y Rappole (1995) argumentan que las comunidades tropicales de aves tienen una dinámica compleja debido al arribo y partida de las especies migratorias que usan diferentes hábitat, lo que produce cambios en la riqueza y composición como respuesta a las distintas escalas espacio-temporales, y a que tales suelen ser más flexibles en sus requerimientos de hábitat y alimento comparado con las especies residentes (Hutto 1992, Villaseñor y Hutto 1995, Ramírez-Albores 2004). A diferencia de las residentes, las cuales necesitan un hábitat más específico, las migratorias son diversas en casi todos los tipos de vegetación (Lynch 1989, Ramírez-Albores 2004).

El inventario de este estudio puede considerarse casi completo y representativo de acuerdo con Gómez de Silva \& Medellín (2001) ya que se registraron especies en las familias o géneros mencionados por estos autores, excepto por la ausencia del género Vireo, así como más de 35 especies y 21 familias. Así mismo, la evidencia que se tuvo de la presencia de las especies registradas al compararlo con inventarios de zonas relativamente cercanas (Nocedal 1981, Rangel-Salazar 1990, González-García 1993, Rangel-Salazar et al. 1993, Warkentin et al. 1995, Greenberg 1996, PueblaOlivares et al. 2002).
Sin embargo, la riqueza de especies estimada para los sitios de estudio es relativamente alta (con excepción del sitio 3, donde se representa el 65\% de la riqueza de especies estimadas) si consideramos que la superficie de estudio no es muy extensa ( $<100 \mathrm{ha}$ ). Se puede argumentar que los resultados aquí presentados dan una referencia para el análisis de comunidades de aves en ambientes similares al de este estudio. No obstante, la riqueza de especies es susceptible de incrementar a través del tiempo, ya que especies accidentales o migratorias de Norteamérica, Centro y Sudamérica pueden presentarse en el área de estudio por la cercanía que tiene con otras zonas o regiones. En este sentido la riqueza reportada en el área de estudio equivale al 91\% de las especies que de acuerdo con el estimador es posible esperar en el área de estudio.

Por otro lado, aunque los sitios de muestreo presentaron los mismos grupos alimenticios, cada uno mostró diferencias en cuanto a su riqueza y composición con el sitio 1 (sitio de muestreo dentro de la REBIMA). Las especies de aves insectívoras presentaron la mayor variación entre una época y otra, disminuyendo de 20 especies observadas en promedio en la época de lluvias a 12 especies en la época de secas. Estos resultados son similares a los encontrados por Verea y Solórzano (1998). Sin embargo, difieren a los obtenidos por Poulin et al. (1994), donde los insectívoros presentaron la menor variación estacional. Las especies insectívoras presentaron la mayor proporción en los distintos sitios de muestreo.

Los insectívoros representados en su mayoría por especies de la familia Tyrannidae fueron más ricos en el sitio 1 , esto puede deberse a la mayor humedad que mantiene a lo largo del año un suministro relativamente constante de invertebrados. Sin embargo, fueron más abundantes en el sitio 3, ambos sitios presentaron una alta similitud en su composición (IS = 0,91). Los insectívoros-frugívoros fueron superiores en riqueza y ligeramente más abundantes en el sitio 1 y 4, siendo ambas muestras similares en composición (IS $=0,85)$. Las especies insectívoras-frugívoras tienden a aumentar en ambientes abiertos, ya que se benefician del aumento en la abundancia de insectos que ocurre durante la época de lluvias y recurren a los frutos al final de la época seca, cuando son más abundantes y los insectos más escasos (Orinas 1969).

Los carnívoros presentaron una riqueza similar en los sitios de muestreo, siendo más abundantes en los sitios abiertos (sitios 2 y 3), donde existe una oferta continúa de alimento (i.e., roedores) por la presencia de pastizales y cultivos. Los nectarívoros fueron ligeramente más ricos y abundantes en los sitios 1 y 4, donde existe una mayor oferta de flores. Sin embargo, Anjos et al. (1997) encuentran que estas especies son más comunes en ambientes abiertos que en áreas arboladas. Probablemente dependan de estos espacios y tengan que moverse a otros lugares en busca de néctar. Los granívoros resultaron más ricos y abundantes en los sitios 2 y 3. Como las selvas tropicales producen 
pocas semillas secas, estas especies están restringidas a la vegetación sucesional (Orinas 1969) que esta presente en los sitios adyacentes a la REBIMA, producto de la tala para el establecimiento de áreas de pastoreo y cultivo (INESemarnap 2000).

Se ha demostrado que la disponibilidad del alimento puede ser un factor que afecta la estructura de las comunidades de aves (Hutto 1985). Poulin et al. (1994), señalan que las poblaciones, gremios o comunidades animales parecen mantener un equilibrio por la limitación temporal de los recursos, lo que implica una relación estrecha entre el número o biomasa de consumidores, su capacidad reproductiva, y el número o biomasa de recursos potencialmente disponibles. Aunque desafortunadamente no se tienen los datos para determinar cual fue la causa real de las diferencias en la riqueza y composición de las comunidades de aves encontradas en los sitios de estudio.

Los resultados mostraron que algunas especies de aves parecen responder de forma diferente al grado de fragmentación y transformación del hábitat, por lo que se presenta una variación en la riqueza y en la composición de las comunidades de aves en los diferentes sitios en el área de estudio (Villard et al. 1999, Ramírez-Albores 2004). Por ejemplo, Sarcoramphus papa, Micrastur semitorquatus, Amazona autumnalis, A. farinosa y Dryocopus lineatus, normalmente habitan en áreas conservadas o con bajo grado de perturbación (i.e., Los Tuxtlas, Veracruz). Sin embargo, estas especies fueron registradas (de uno a cinco individuos por muestreo/mensual) alimentándose o en busca de alimento en los sitios adyacentes a la REBIMA, utilizando a estos sitios como hábitat sumidero y al sitio 1 (sitio dentro de la REBIMA) como hábitat fuente (Pulliam \& Danielson 1991). Probablemente, la disminución de los recursos alimentarios determina que muchas especies deban aumentar su área y horas de actividad en busca de alimento (Karr et al. 1982) además, que muchas especies se ven en la necesidad de cambiar su dieta y tengan que buscar alimento en lugares que antes no frecuentaban (Verea \& Solórzano 1998).

El área de estudio puede ser considerada un área rica en especies de aves a pesar de que esta siendo perturbada por diversas actividades antropogénicas. Es necesario que se realicen otros muestreos, abarcando una mayor área de muestreo (en este estudio fue $<100$ ha) y otros tipos de vegetación, lo que permitiría tener una mejor representatividad de la riqueza avifaunística del área. Las áreas de estudio contribuyen en el mantenimiento de la riqueza y diversidad de especies en la zona. Podría argumentarse que la incorporación de parches de vegetación con distintas fases de regeneración natural, de formas y tamaños variables podría ser necesaria para la supervivencia y reproducción de muchas especies de aves (Woinarski et al. 1992). Para asegurar la continuidad de tales especies, en primer lugar, se requiere la protección y conservación de los hábitats primarios y la implementación de estudios sobre la biología básica de las poblaciones que permitan, a través del conocimiento obtenido, plantear alternativas y estrategias para su adecuado uso, sin poner en riesgo la estabilidad del ecosistema tropical. Es necesario también mantener algunos hábitat sucesionales, los cuales son usados como sitios de forrajeo por una amplia variedad de especies residentes y migratorias, con el fin de mantener la diversidad avifaunística.

\section{Agradecimientos}

A El Colegio de la Frontera Sur por su apoyo para la realización del presente estudio. Al LAIGE (ECOSUR-San Cristóbal) por el apoyo en la realización del mapa del área de estudio. A todas las personas que participaron directa o indirectamente en la realización del presente estudio, en especial a Guadalupe Ramírez. A Raúl Ortiz-Pulido por sus valiosos comentarios. Los comentarios y/o sugerencias de revisores anónimos que ayudaron a mejorar el contenido de este estudio.

\section{Referencias}

ANDRADE, G.I. \& RUBIO, H. 1994. Sustainable use of the tropical rainforest: evidence from the avifauna in a shifting-cultivation habitat mosaic in the Colombian Amazon. Conserv. Biol. 8: 545-554.

ANJOS, L. DOS, SCHUCHMANN, K.L.\&BERNDT, R. 1997. Avifaunal composition, species richness, and status in the Tibagi River Basin, Parana State, Southern Brazil. Ornitol. Neotrop. 8: 145-173.

A.O.U. (AMERICAN ORNITHOLOGISTS’ UNION). 1998. Check-list of North American birds. 7 ed. Allen Press, Washington, D. C.

A.O.U. (AMERICAN ORNITHOLOGISTS' UNION). 2000. Forty-second supplement to the American Ornithologists' Union check-list of North American birds. Auk 117: 847-858.

ARIZMENDI, M.C., BERLANGA, H., MÁRQUEZVALDELAMAR, L., NAVARIJO, L. \& ORNELAS, J.F. 1990. Avifauna de la región de Chamela, Jalisco. Cuadernos del Instituto de Biología No. 4, Universidad Nacional Autónoma de México, México.

BANKS, R.C., CICERO, C., DUNN, J.L., KRATTER, A.W., RASMUSSEN, P.C., REMSEN JR., J.V., RISING, J.D. \& STOTZ, D.F. 2002. Forty-third supplement to the American Ornithologists' Union check-list of North American birds. Auk 119: 897-906.

BANKS, R.C., CICERO, C., DUNN, J.L., KRATTER, A.W., RASMUSSEN, P.C., REMSEN JR., J.V., RISING, J.D. \& STOTZ, D.F. 2003. Forty-fourth supplement to the American Ornithologists' Union check-list of North American birds. Auk 120: 923-931. 
BANKS, R.C., CICERO, C., DUNN, J.L., KRATTER, A.W., RASMUSSEN, P.C., REMSEN JR., J.V., RISING, J.D. \& STOTZ, D.F. 2004. Forty-fifth supplement to the American Ornithologists' Union check-list of North American birds. Auk 121: 985-995.

BANKS, R.C., CICERO, C., DUNN, J.L., KRATTER, A.W., RASMUSSEN, P.C., REMSEN JR., J.V., RISING, J.D. \& STOTZ, D.F. 2005. Forty-sixth supplement to the American Ornithologists' Union check-list of North American birds. Auk 122: 1026-1031.

BLAKE, J.G. \& LOISELLE, B.A. 1991. Variation in resource abundance affects capture rates of birds in three lowland habitats in Costa Rica. Auk 108: 114-130.

BLAKE, J.G. \& LOISELLE, B.A. 2001. Birds assemblages in second-growth and old-growth forest, Costa Rica: perspectives from mist nest and point counts. Auk 118: 304-326.

BLAKE, J.G., STILES, F.G. \& LOISELLE, B.A. 1990. Birds of La Selva Biological Station: Habitat, use, trophic composition, and migrants. In Four Neotropical rainforests. (A.H. Gentry, ed.). Yale University Press, New Haven, p. 161-182.

BOJORGES, B. J. C. \& LÓPEZ-MATA, L. 2001. Abundancia y distribución temporal de aves en una selva mediana subperennifolia en el centro de Veracruz, México. An. Inst. Biol. Univ. Nac. Auton. Mex. Ser. Zool. 72: 259-283.

BOJORGES, B. J. C. \& LÓPEZ-MATA, L. 2005. Riqueza y diversidad de especies de aves en una selva mediana subperennifolia en el centro de Veracruz, México. Acta Zool. Mex. 21: 1-20.

BUSKIRK, W.H. 1980. Influence of meteorological patterns and Trans-Gulf migration on the calendars of latitudinal migrants. In Migrant birds in the neotropics. Ecology, behavior, and conservation. (A. Keast \& E.S. Morton, eds.). Symposia of the National Zoological Park. Smithsonian Institution Press, Washington, D.C., p. 485-491.

CASTILLO-CAMPOS, G. \& NARAVE, H. 1992. Contribución al conocimiento de la vegetación de la Reserva de la Biosfera Montes Azules, Selva Lacandona, Chiapas, México. In Reserva de la Biosfera Montes Azules: Investigación para su conservación. (M.A. VázquezSánchez \& M.A. Ramos, eds.). Publicación Especial Ecosfera, México, p. 51-85.

COLWELL, R.K. \& CODDINGTON, J.A. 1994. Estimating terrestrial biodiversity through extrapolation. Philos. Tr. R. Soc. B. 345: 101-118.

EMLEN, J.T. 1971. Population densities of birds derived from transect counts. Auk 88: 323-342.

ESTRADA, A., COATES-ESTRADA, R. \& MERITT, D.A. 1997. Anthropogenic landscape changes and avian diversity at Los Tuxtlas, Mexico. Biodivers. Conserv. 6: 19-43.
ESTRADA, A., CAMMARANO, P. \& COATES-ESTRADA, R. 2000. Bird species richness in vegetation fences and in strips of residual rain forest vegetation at Los Tuxtlas, Mexico. Biol. Conserv. 9: 1399-1416.

GÓMEZ DE SILVA, H. \& MEDELLÍN, R.A. 2001. Evaluating completeness of species list for conservation and macroecology: A case study of Mexican land birds. Conserv. Biol. 15: 1384-1395.

GONZÁLEZ-GARCÍA, F. 1993. Avifauna de la Reserva de la Biosfera “Montes Azules”, Selva Lacandona, Chiapas, México. Acta Zool. Mex. 55: 1-86.

GREENBERG, R. 1986. Competition in migrant birds in the nonbreeding season. Curr. Ornithol. 3: 281-303.

GREENBERG, R. 1996. Managed forest patches and the diversity of birds in southern Mexico. In Forest patches in tropical landscapes. (J. Schelhas \& R. Greenberg, eds.). Island Press, Washington, D.C., p. 59-90.

HESPENHEIDE, H.A. 1980. Bird community structure in two Panama forests: resident, migrants, and seasonality during the nonbreeding season. In Migrant birds in the neotropics. Ecology, behavior, and conservation. (A. Keast \& E.S. Morton, eds.). Symposia of the National Zoological Park. Smithsonian Institution Press, Washington, D.C., p. 227-237.

HUTTO, R.L. 1985. Habitat selection by nonbreeding, migratory land birds. In Habitat selection in birds. (M.L. Cody, ed.). Academic Press, San Diego, p. 455-476.

HUTTO, R.L. 1992. Habitat distributions of migratory landbird species in western Mexico. In Ecology and conservation of neotropical migrant landbirds. (J. Hagan \& D. Johnston, eds.). Smithsonian Institution Press, Washington, D.C., p. 221-23.

HOWELL, S.N. \& WEBB, S. 1995. A guide to the birds of Mexico and northern Central America. Oxford University Press, New York.

INE-Semarnap. 2000. Programa de manejo de la Reserva de la Biosfera Montes Azules. Instituto Nacional de Ecología-Secretaría del Medio Ambiente y Recursos Naturales (INE-Semarnap), México.

KARR, J.R. 1977. Ecological correlates of rarity in a tropical forest birds community. Auk 94: 240-247.

KARR, J.R. 1990. The avifauna of Barro Colorado Island and the Pipeline Roas, Panama. In Four Neotropical rainforests. (A. H. Gentry, ed.). Yale University Press, New Haven, p. 183-198.

KARR, J.R., SCHEMSKE, D.W. \& BROKAW, N.V.L. 1982. Temporal variation in the understory bird community of a tropical forest. In The ecology of a tropical forest. (E.G. Leigh Jr., A.S. Rand \& D.M. Windsor, eds.). Smithsonian Institution Press, Washington, D. C., p. 441-453. 
LEVEY, D.J. 1988. Spatial and temporal variation in Costa Rican fruit and fruit-eating bird abundance. Ecol. Monogr. 58: 257-269.

LOISELLE, B.A. \& BLAKE, J.G. 1992. Population variation in a tropical bird community. Bioscience 11: 838-845.

LÓPEZ DE CASENAVE, L. \& MARONE, L. 1996. Efectos de la riqueza y la equitatividad sobre los valores de diversidad en comunidades de aves. Ecología 10: 447-455.

LORD, J. M. \& NORTON, D.A. 1990. Scale and the spatial concept of fragmentation. Conserv. Biol. 4: 197-202.

LYNCH, J.F. 1989. Distribution of overwintering neartic migrants in the Yucatan Peninsula, II: Use of relative and human-modified vegetation. In Ecology and conservation of neotropical migrant landbirds. (J.M. Hagan \& D.W. Johnston, eds.). Smithsonian Institution Press, Washington, D.C., p. 178-196.

MILLS, E.D. \& ROGERS, D.T. 1990. Neartic passerine fall migration in central Belize. Wilson Bull. 102: 146-150.

MIRANDA, F. \& HERNÁNDEZ, X.E. 1963. Los tipos de vegetación de México y su clasificación. Bol. Soc. Bot. Mex. 28: 29-179.

McINTYRE, N. 1995. Effects on forest match size on avian diversity. Landscape Ecol. 10: 85-99.

NOCEDAL, J. 1981. Avifauna de la región LacanjáChansayab, Selva Lacandona, Chiapas. In Estudios ecológicos en el trópico mexicano. (P. Reyes-Castillo, ed.). Instituto de Ecología A. C., México, p. 11-41.

ORINAS, G.H. 1969. The number of bird species in some tropical forests. Ecology 50: 783-801.

ORTIZ-PULIDO, R. 1994. Frugivoría y dispersión de semillas por aves en el Moro la Mancha, Veracruz. Tesis de licenciatura, Universidad Veracruzana, Xalapa, México.

PETERSON, R.T. \& CHALIF, E.L. 1994. Aves de México: Guía de campo. Edit. Diana, México.

PETIT, D.R., LYNCH, J.F., HUTTO, R.L., BLAKE, J.G. \& WAIDE, R.B. 1995. Habitat use and conservation in the Neotropics. In Ecology and management of Neotropical migratory birds: a synthesis and review of critical issues. (T.E. Martin \& D.M. Finch, eds.). Oxford University Press, New York, p. 145-197.

POULIN, B., LEFEBVRE, G. \& MCNEIL, R. 1994. Characteristics of feeding guilds and variation in diets of birds species of three tropical sites. Biotropica 26: 187-197.

PUEBLA-OLIVARES, F., RODRIGUEZ-AYALA, E., HERNÁNDEZ-BAÑOS, B. \& NAVARRO, A. 2002. Status and conservation of the avifauna of the Yaxchilán Natural Monument, Chiapas, Mexico. Ornitol. Neotrop. 13:381-396.

PULLIAM, H.R. \& DANIELSON, B.J. 1991. Sources, sinks, and habitat selection: A landscape perspective on population dynamics. Am. Nat. 137: S50-S66.
RAMÍREZ-ALBORES, J.E. 2004. Efecto de la estructura del paisaje sobre la diversidad a, b y g de comunidades de aves de San Fernando, Chiapas, México. Tesis de Maestría, El Colegio de la Frontera Sur, San Cristóbal de las Casas, México.

RAMOS, M.A. 1985. Endangered tropical birds in Mexico and northern Central America. In Conservation of tropical forest birds. (A.W. Diamond \& T.E. Lovejoy, eds.). ICBP, Technical Publication No. 4, Cambridge, p. 305-318.

RANGEL-SALAZAR, J. 1990. Abundancia y diversidad en una comunidad de aves en la Reserva de la Biosfera Montes Azules, Selva Lacandona, Chiapas. Tesis de licenciatura, Universidad Nacional Autónoma de México. México.

RANGEL-SALAZAR, J.L., ENRÍQUEZ-ROCHA, P.L. \& VEGA-RIVERA, J.H. 1993. Riqueza de especies de aves de sotobosque en la Selva Lacandona, Chiapas, México. Rev. Biol. Trop. 41: 273-279.

RAPPOLE, J.H. 1995. The ecology of migrant birds: A Neotropical perspective. Smithsonian Institution Press, Washington, D.C.

RAVINOVICH, J. 1981. Introducción a la ecología de poblaciones animales. Edit. Continental, México.

ROBINSON, S.K. \& TERBORGH, J. 1997. Bird community dynamics along primary successional gradients o fan Amazonian Whitewater River. Ornithol. Monogr. 48: 641-672.

ROBINSON, W.D., BRAWN, J.D. \& ROBINSON, S.K. 2000. Forest bird community structure in Central Panama: Influence of spatial scale and biogeography. Ecol. Monogr. 70: 209-235.

SMITH, A.L., SALGADO, J. \& ROBERTSON, R.J. 2001. Distribution patterns of migrant and resident birds in successional forest of the Yucatan Peninsula, Mexico. Biotropica 33: 153-170.

SOBERÓN, J. \& LLORENTE, J. 1993. The use of species accumulation functions for the prediction of species richness. Conserv. Biol. 7: 480-488.

STAICER, C.A. 1992. Social behavior of the Northern Parula, Cape May Warbler, and Prairie Warbler wintering in second-growth forest in southerwestern Puerto Rico. In Ecology and conservation of Neotropical migrant landbirds. (M. Hagan \& D.W. Johnston, eds.). Smithsonian Institution Press, Washington, D. C., p. 308-320.

STILES, F.G. 1983. Check-list of birds. In Costa Rican natural history (D.H. Janzen, ed.). University of Chicago Press, Chicago, p. 502-543.

TERBORGH, J. \& WESKE, J.S. 1969. Colonization of secondary habitats by Peruvian birds. Ecology 50: 765-782.

VEREA, C. \& SOLÓRZANO, A. 1998. La avifauna del sotobosque de una selva decidua tropical en Venezuela. Ornitol. Neotrop. 9: 161-176. 
VEREA, C., FERNÁNDEZ-BADILLO, D. \& SOLÓRZANO, A. 2000. Variación en la composición de las comunidades de aves de sotobosque de dos bosques en el norte de Venezuela. Ornitol. Neotrop. 11: 65-79.

VILLARD, M., TRZCINSKI, M.K. \& MERRIAM, G. 1999. Fragmentation effects on forest birds: relative influence of woodland cover and configuration on landscape occupancy. Conserv. Biol. 13: 774-783.

VILLASEÑOR, J.F. \& HUTTO, R.L. 1995. The importance of agricultural areas for the conservation of neotropical migratory landbirds in Western Mexico. In Conservation of neotropical migratory birds in Mexico. (M.H. Wilson \& S.A. Sader, eds.). Miscellaneous Publication. Agricultural and Forest Experiment Station. Maine, p. 59-80.

WARKENTIN, I.G, GREENBERG, R. \& SALGADO, J. 1995. Songbird use of gallery woodlands in recently cleared and older settled landscapes of the Selva Lacandona, Chiapas, Mexico. Conserv. Biol. 9: 1095-1106.

WIENS, J.A. 1997. Metapopulation dynamics and landscape ecology. In Metapopulation biology. (I.A. Hanski \& M.E. Gilpin, eds.). Academic Press, New York, p. 43-62.

WIENS, J.A. \& ROTEMBERRY, J. T. 1981. Habitat associations and community structure of birds in shrubsteppe environments. Ecol. Monogr. 51: 21-41.

WILLIS, E.O. 1980. Ecological roles of migratory and resident birds on Barro Colorado Island, Panama. In Migrant birds in the Neotropics: Ecology, behavior, distribution and conservation. (A. Keast \& E.S. Morton, eds.). Smithsonian Institution Press, Washington, D. C., p. 205-225.

WINKER, K., OEHLENSCHLAGER, R.J., RAMOS, M.A., ZINK, R.M., RAPPOLE, J.H. \& WARNER, D.W. 1992. Avian distribution and abundance records for the Sierra de Los Tuxtlas, Veracruz, Mexico. Wilson Bull. 104: 699-718.

WOINARSKI, J.C.Z., WHITEHEAD, P.J., BOWMAN, D.M. \& RUSELL-SMITH, J. 1992. Conservation of mobile species in a variable environment: the problem of reserve design in the Northern Territory, Australia. Global Ecol. Biogeogr. Lett. 2: 1-10.

WOLDA, H. 1990. Food availability for an insectivore and how to measure it. In Avian foraging: Theory, methodology, and applications. (M. L. Morrison, ed.). Studies in Avian Biology 13, p. 38-143.
Título: Variación en la composición de comunidades de aves en la Reserva de la Biosfera Montes Azules y áreas adyacentes, Chiapas, México

Autor: Ramírez-Albores, J.E.

Biota Neotropica, Vol. 6 (número 2): 2006

http://www.biotaneotropica.org.br/v6n2/pt/ abstract?article+bn02806022006

Recebido em 09/09/2005 - Versão Reformulada recebida em 25/05/2006 - Publicado em 28/06/2006

ISSN 1676-0603 
Apéndice 1. Listado de la avifauna registrada en sitios de muestreo en la Reserva de la Biosfera Montes Azules y áreas adyacentes, Chiapas, México.

Appendix 1. List of the registered avifauna in the study sites in the Biosphere Reserve Montes Azules and adjacent areas, Chiapas, Mexico.

\begin{tabular}{|c|c|c|c|c|c|c|}
\hline Especie & Sitio 1 & Sitio 2 & Sitio 3 & Sitio 4 & Estatus & Dieta \\
\hline Tinamus major & $\mathrm{x}$ & & $\mathrm{x}$ & $\mathrm{x}$ & $\mathrm{R}$ & $\mathrm{O}$ \\
\hline Crypturellus cinnamomeus & $\mathrm{x}$ & & & $\mathrm{x}$ & $\mathrm{R}$ & $\mathrm{O}$ \\
\hline Dendrocygna autumnalis & $\mathrm{x}$ & $\mathrm{x}$ & & & $\mathrm{R}$ & $\mathrm{O}$ \\
\hline Anas discors & $\mathrm{x}$ & $\mathrm{x}$ & & & $\mathrm{W}$ & $\mathrm{O}$ \\
\hline Ortalis vetula & $\mathrm{x}$ & & $\mathrm{x}$ & $\mathrm{x}$ & $\mathrm{R}$ & GF \\
\hline Penelope purpurascens & $\mathrm{x}$ & & & $\mathrm{x}$ & $\mathrm{R}$ & GF \\
\hline Phalacrocorax brasilianus & $\mathrm{x}$ & & & & $\mathrm{R}$ & $\mathrm{C}$ \\
\hline Tigrisoma mexicanum & $\mathrm{x}$ & $\mathrm{x}$ & & & $\mathrm{R}$ & $\mathrm{C}$ \\
\hline Ardea herodias & $\mathrm{x}$ & & & & W & $\mathrm{C}$ \\
\hline Ardea alba & $\mathrm{x}$ & $\mathrm{x}$ & & & $\mathrm{W}$ & $\mathrm{C}$ \\
\hline Egretta thula & $\mathrm{x}$ & $\mathrm{x}$ & & & $\mathrm{W}$ & $\mathrm{CI}$ \\
\hline Egretta caerulea & $\mathrm{x}$ & $\mathrm{x}$ & & & $\mathrm{W}$ & $\mathrm{CI}$ \\
\hline Bubulcus ibis & & $\mathrm{x}$ & $\mathrm{x}$ & & $\mathrm{R}$ & I \\
\hline Agamia agami & $\mathrm{x}$ & $\mathrm{x}$ & & & $\mathrm{R}$ & CI \\
\hline Coragyps atratus & $\mathrm{x}$ & $\mathrm{x}$ & $\mathrm{x}$ & $\mathrm{x}$ & $\mathrm{R}$ & $\mathrm{C}$ \\
\hline Cathartes aura & $\mathrm{x}$ & $\mathrm{x}$ & $\mathrm{x}$ & $\mathrm{x}$ & $\mathrm{R}$ & $\mathrm{C}$ \\
\hline Sarcoramphus papa & $\mathrm{x}$ & & $\mathrm{x}$ & & $\mathrm{R}$ & $\mathrm{C}$ \\
\hline Leptodon cayanensis & $\mathrm{x}$ & $\mathrm{x}$ & & $\mathrm{x}$ & $\mathrm{R}$ & $\mathrm{C}$ \\
\hline Elanus leucurus & & $\mathrm{x}$ & $\mathrm{x}$ & & $\mathrm{R}$ & $\mathrm{C}$ \\
\hline Busarellus nigricollis & $\mathrm{x}$ & $\mathrm{x}$ & & & $\mathrm{R}$ & $\mathrm{C}$ \\
\hline Accipiter striatus & $\mathrm{x}$ & $\mathrm{x}$ & & $\mathrm{x}$ & $\mathrm{W}$ & $\mathrm{C}$ \\
\hline Leucopternis albicollis & $\mathrm{x}$ & & & $\mathrm{x}$ & $\mathrm{R}$ & $\mathrm{C}$ \\
\hline Asturina nitida & $\mathrm{x}$ & $\mathrm{x}$ & & $\mathrm{x}$ & $\mathrm{R}$ & $\mathrm{C}$ \\
\hline Buteogallus anthracinus & $\mathrm{x}$ & $\mathrm{x}$ & $\mathrm{x}$ & $\mathrm{x}$ & $\mathrm{R}$ & $\mathrm{C}$ \\
\hline Buteo magnirostris & $\mathrm{x}$ & & & $\mathrm{x}$ & $\mathrm{R}$ & $\mathrm{C}$ \\
\hline Buteo jamaicensis & $\mathrm{x}$ & $\mathrm{x}$ & & $\mathrm{x}$ & $\mathrm{R}$ & $\mathrm{C}$ \\
\hline Micrastur ruficollis & & $\mathrm{x}$ & $\mathrm{x}$ & & $\mathrm{R}$ & $\mathrm{C}$ \\
\hline Micrastur semitorquatus & $\mathrm{x}$ & $\mathrm{x}$ & $\mathrm{x}$ & $\mathrm{x}$ & $\mathrm{R}$ & $\mathrm{C}$ \\
\hline Falco rufigularis & & $\mathrm{x}$ & $\mathrm{x}$ & & $\mathrm{R}$ & $\mathrm{C}$ \\
\hline Himantopus mexicanus & $\mathrm{x}$ & $\mathrm{x}$ & & & $\mathrm{T}$ & I \\
\hline Jacana spinosa & $\mathrm{x}$ & $\mathrm{x}$ & & & $\mathrm{R}$ & I \\
\hline
\end{tabular}


Apéndice 1. continuación

\begin{tabular}{|c|c|c|c|c|c|c|}
\hline Especie & Sitio 1 & Sitio 2 & Sitio 3 & Sitio 4 & Estatus & Dieta \\
\hline Actitis macularius & $\mathrm{x}$ & & & & $\mathrm{W}$ & I \\
\hline Patagioenas cayennensis & $\mathrm{x}$ & & $\mathrm{x}$ & $\mathrm{x}$ & $\mathrm{R}$ & GF \\
\hline Patagioenas nigrirostris & $\mathrm{x}$ & & & $\mathrm{x}$ & $\mathrm{R}$ & GF \\
\hline Columbina inca & & $\mathrm{x}$ & $\mathrm{x}$ & & $\mathrm{R}$ & G \\
\hline Columbina passerina & & $\mathrm{x}$ & $\mathrm{x}$ & & $\mathrm{R}$ & G \\
\hline Columbina talpacoti & & $\mathrm{x}$ & $\mathrm{x}$ & $\mathrm{x}$ & $\mathrm{R}$ & G \\
\hline Claravis pretiosa & & $\mathrm{x}$ & $\mathrm{x}$ & & $\mathrm{R}$ & G \\
\hline Leptotila verreauxi & $\mathrm{x}$ & & $\mathrm{x}$ & $\mathrm{x}$ & $\mathrm{R}$ & GF \\
\hline Aratinga nana & $\mathrm{x}$ & $\mathrm{x}$ & & $\mathrm{x}$ & $\mathrm{R}$ & GF \\
\hline Ara macao & $\mathrm{x}$ & $\mathrm{x}$ & $\mathrm{x}$ & $\mathrm{x}$ & $\mathrm{R}$ & GF \\
\hline Pionopsitta haematotis & $\mathrm{x}$ & & $\mathrm{x}$ & $\mathrm{x}$ & $\mathrm{R}$ & GF \\
\hline Amazona autumnalis & $\mathrm{x}$ & $\mathrm{x}$ & $\mathrm{x}$ & $\mathrm{x}$ & $\mathrm{R}$ & GF \\
\hline Amazona farinosa & $\mathrm{x}$ & $\mathrm{x}$ & $\mathrm{x}$ & $\mathrm{x}$ & $\mathrm{R}$ & GF \\
\hline Crotophaga sulcirostris & $\mathrm{x}$ & $\mathrm{x}$ & $\mathrm{x}$ & $\mathrm{x}$ & $\mathrm{R}$ & GIF \\
\hline Megascops guatemalae & $\mathrm{x}$ & & & $\mathrm{x}$ & $\mathrm{R}$ & $\mathrm{C}$ \\
\hline Glaucidium brasilianum & $\mathrm{x}$ & $\mathrm{x}$ & & $\mathrm{x}$ & $\mathrm{R}$ & $\mathrm{C}$ \\
\hline Chordeiles acutipennis & $\mathrm{x}$ & $\mathrm{x}$ & & $\mathrm{x}$ & W & I \\
\hline Streptoprocne zonaris & & $\mathrm{x}$ & $\mathrm{x}$ & & $\mathrm{R}$ & I \\
\hline Chaetura vauxi & $\mathrm{x}$ & $\mathrm{x}$ & $\mathrm{x}$ & $\mathrm{x}$ & $\mathrm{R}$ & I \\
\hline Phaethornis longirostris & $\mathrm{x}$ & $\mathrm{x}$ & & $\mathrm{x}$ & $\mathrm{R}$ & $\mathrm{N}$ \\
\hline Phaethornis striigularis & $\mathrm{x}$ & & $\mathrm{x}$ & $\mathrm{x}$ & $\mathrm{R}$ & $\mathrm{N}$ \\
\hline Abeillia abeillei & $\mathrm{x}$ & $\mathrm{x}$ & & $\mathrm{x}$ & $\mathrm{R}$ & $\mathrm{N}$ \\
\hline Amazilia beryllina & $\mathrm{x}$ & & & $\mathrm{x}$ & $\mathrm{R}$ & $\mathrm{N}$ \\
\hline Amazilia tzacatl & $\mathrm{x}$ & $\mathrm{x}$ & $\mathrm{x}$ & $\mathrm{x}$ & $\mathrm{R}$ & $\mathrm{N}$ \\
\hline Trogon violaceus & $\mathrm{x}$ & & $\mathrm{x}$ & $\mathrm{x}$ & $\mathrm{R}$ & IF \\
\hline Momotus momota & $\mathrm{x}$ & & $\mathrm{x}$ & $\mathrm{x}$ & $\mathrm{R}$ & CIF \\
\hline Ceryle torquatus & $\mathrm{x}$ & $\mathrm{x}$ & & & $\mathrm{R}$ & $\mathrm{C}$ \\
\hline Chloroceryle americana & $\mathrm{x}$ & & & $\mathrm{x}$ & $\mathrm{R}$ & $\mathrm{CI}$ \\
\hline Malacoptila panamensis & $\mathrm{x}$ & & & & $\mathrm{R}$ & I \\
\hline Galbula ruficauda & $\mathrm{x}$ & & & & $\mathrm{R}$ & I \\
\hline Pteroglossus torquatus & $\mathrm{x}$ & & $\mathrm{x}$ & $\mathrm{x}$ & $\mathrm{R}$ & GF \\
\hline Ramphastos sulfuratus & $\mathrm{x}$ & & $\mathrm{x}$ & $\mathrm{x}$ & $\mathrm{R}$ & GF \\
\hline Melanerpes pucherani & $\mathrm{x}$ & & $\mathrm{x}$ & $\mathrm{x}$ & $\mathrm{R}$ & I \\
\hline Melanerpes aurifrons & $\mathrm{x}$ & $\mathrm{x}$ & $\mathrm{x}$ & $\mathrm{x}$ & $\mathrm{R}$ & IF \\
\hline
\end{tabular}


Apéndice 1. continuación

\begin{tabular}{|c|c|c|c|c|c|c|}
\hline Especie & Sitio 1 & Sitio 2 & Sitio 3 & Sitio 4 & Estatus & Dieta \\
\hline Dryocopus lineatus & $\mathrm{x}$ & $\mathrm{x}$ & $\mathrm{x}$ & $\mathrm{x}$ & $\mathrm{R}$ & $\mathrm{I}$ \\
\hline Campephilus guatemalensis & $\mathrm{x}$ & & $\mathrm{x}$ & $\mathrm{x}$ & $\mathrm{R}$ & I \\
\hline Xiphocolaptes promeropirhynchus & $\mathrm{x}$ & & $\mathrm{x}$ & $\mathrm{x}$ & $\mathrm{R}$ & I \\
\hline Dendrocolaptes certhia & $\mathrm{x}$ & & $\mathrm{x}$ & $\mathrm{x}$ & $\mathrm{R}$ & I \\
\hline Xiphorhynchus flavigaster & $\mathrm{x}$ & & & $\mathrm{x}$ & $\mathrm{R}$ & I \\
\hline Lepidocolaptes souleyetii & $\mathrm{x}$ & $\mathrm{x}$ & $\mathrm{x}$ & $\mathrm{x}$ & $\mathrm{R}$ & I \\
\hline Taraba major & $\mathrm{x}$ & & & $\mathrm{x}$ & $\mathrm{R}$ & I \\
\hline Thamnophilus doliatus & $\mathrm{x}$ & & $\mathrm{x}$ & $\mathrm{x}$ & $\mathrm{R}$ & I \\
\hline Cercomacra tyrannina & $\mathrm{x}$ & & $\mathrm{x}$ & $\mathrm{x}$ & $\mathrm{R}$ & I \\
\hline Formicarius analis & $\mathrm{x}$ & & & & $\mathrm{R}$ & I \\
\hline Leptopogon amaurocephalus & $\mathrm{x}$ & & & & $\mathrm{R}$ & I \\
\hline Poecilotriccus sylvia & $\mathrm{x}$ & & & $\mathrm{x}$ & $\mathrm{R}$ & I \\
\hline Contopus cinereus & $\mathrm{x}$ & & $\mathrm{x}$ & & $\mathrm{R}$ & I \\
\hline Empidonax minimus & $\mathrm{x}$ & $\mathrm{x}$ & & & W & I \\
\hline Empidonax fulvifrons & $\mathrm{x}$ & & & & $\mathrm{R}$ & I \\
\hline Rhytipterna holerythra & $\mathrm{x}$ & & & $\mathrm{x}$ & $\mathrm{R}$ & I \\
\hline Myiarchus tyrannulus & & $\mathrm{x}$ & $\mathrm{x}$ & $\mathrm{x}$ & $\mathrm{R}$ & IF \\
\hline Pitangus sulphuratus & $\mathrm{x}$ & $\mathrm{x}$ & & $\mathrm{x}$ & $\mathrm{R}$ & $\mathrm{O}$ \\
\hline Megarhynchus pitangua & $\mathrm{x}$ & & $\mathrm{x}$ & $\mathrm{x}$ & $\mathrm{R}$ & IF \\
\hline Myozetetes similis & $\mathrm{x}$ & $\mathrm{x}$ & & $\mathrm{x}$ & $\mathrm{R}$ & IF \\
\hline Myiodynastes luteiventris & $\mathrm{x}$ & & $\mathrm{x}$ & & $\mathrm{S}$ & IF \\
\hline Tyrannus melancholicus & $\mathrm{x}$ & $\mathrm{x}$ & $\mathrm{x}$ & $\mathrm{x}$ & $\mathrm{R}$ & I \\
\hline Tyrannus savanna & & $\mathrm{x}$ & & & $\mathrm{R}$ & I \\
\hline Lipaugus unirufus & $\mathrm{x}$ & & & $\mathrm{x}$ & $\mathrm{R}$ & I \\
\hline Tityra semifasciata & $\mathrm{x}$ & $\mathrm{x}$ & $\mathrm{x}$ & & $\mathrm{R}$ & IF \\
\hline Manacus candei & $\mathrm{x}$ & & & & $\mathrm{R}$ & I \\
\hline Cyclarhis gujanensis & $\mathrm{x}$ & & & $\mathrm{x}$ & $\mathrm{R}$ & I \\
\hline Cyanocorax yncas & $\mathrm{x}$ & & & $\mathrm{x}$ & $\mathrm{R}$ & IF \\
\hline Cyanocorax morio & $\mathrm{x}$ & $\mathrm{x}$ & $\mathrm{x}$ & $\mathrm{x}$ & $\mathrm{R}$ & IF \\
\hline Progne chalybea & $\mathrm{x}$ & $\mathrm{x}$ & $\mathrm{x}$ & $\mathrm{x}$ & $\mathrm{S}$ & $\mathrm{I}$ \\
\hline Tachycineta albilinea & $\mathrm{x}$ & & & $\mathrm{x}$ & $\mathrm{R}$ & I \\
\hline Thryothorus maculipectus & $\mathrm{x}$ & $\mathrm{x}$ & $\mathrm{x}$ & $\mathrm{x}$ & $\mathrm{R}$ & I \\
\hline Uropsila leucogastra & $\mathrm{x}$ & & $\mathrm{x}$ & $\mathrm{x}$ & $\mathrm{R}$ & I \\
\hline Ramphocaenus melanurus & $\mathrm{x}$ & & $\mathrm{x}$ & $\mathrm{x}$ & $\mathrm{R}$ & I \\
\hline
\end{tabular}

http://www.biotaneotropica.org.br 
Apéndice 1. continuación

\begin{tabular}{|c|c|c|c|c|c|c|}
\hline Especie & Sitio 1 & Sitio 2 & Sitio 3 & Sitio 4 & Estatus & Dieta \\
\hline Polioptila caerulea & $\mathrm{x}$ & $\mathrm{x}$ & $\mathrm{x}$ & $\mathrm{x}$ & W & I \\
\hline Turdus grayi & $\mathrm{x}$ & & $\mathrm{x}$ & $\mathrm{x}$ & $\mathrm{R}$ & IF \\
\hline Dumetella carolinensis & $\mathrm{x}$ & & & $\mathrm{x}$ & W & IF \\
\hline Vermivora peregrina & $\mathrm{x}$ & & & $\mathrm{x}$ & W & I \\
\hline Vermivora ruficapilla & $\mathrm{x}$ & & & $\mathrm{x}$ & W & IF \\
\hline Dendroica magnolia & $\mathrm{x}$ & $\mathrm{x}$ & & $\mathrm{x}$ & W & IF \\
\hline Mniotilta varia & $\mathrm{x}$ & $\mathrm{x}$ & & $\mathrm{x}$ & W & I \\
\hline Setophaga ruticilla & & $\mathrm{x}$ & $\mathrm{x}$ & & W & I \\
\hline Helmitheros vermivorum & $\mathrm{x}$ & & & & W & I \\
\hline Limnothlypis swainsonii & $\mathrm{x}$ & & & & $\mathrm{T}$ & I \\
\hline Seiurus aurocapillus & $\mathrm{x}$ & & & $\mathrm{x}$ & W & I \\
\hline Wilsonia pusilla & & $\mathrm{x}$ & $\mathrm{x}$ & & W & I \\
\hline Myioborus miniatus & $\mathrm{x}$ & & & $\mathrm{x}$ & $\mathrm{R}$ & I \\
\hline Granatellus sallaei & $\mathrm{x}$ & & & & $\mathrm{R}$ & I \\
\hline Habia rubica & $\mathrm{x}$ & & & $\mathrm{x}$ & $\mathrm{R}$ & IF \\
\hline Piranga rubra & $\mathrm{x}$ & $\mathrm{x}$ & $\mathrm{x}$ & $\mathrm{x}$ & $\mathrm{W}$ & IF \\
\hline Ramphocelus sanguinolentus & $\mathrm{x}$ & & & $\mathrm{x}$ & $\mathrm{R}$ & GF \\
\hline Ramphocelus passerinii & & & & $\mathrm{x}$ & $\mathrm{R}$ & GF \\
\hline Thraupis episcopus & & & $\mathrm{x}$ & & $\mathrm{R}$ & IF \\
\hline Thraupis abbas & $\mathrm{x}$ & & & $\mathrm{x}$ & $\mathrm{R}$ & IF \\
\hline Cyanerpes cyaneus & $\mathrm{x}$ & & & $\mathrm{x}$ & $\mathrm{R}$ & IN \\
\hline Volatinia jacarina & $\mathrm{x}$ & $\mathrm{x}$ & & $\mathrm{x}$ & $\mathrm{R}$ & G \\
\hline Sporophila torqueola & & $\mathrm{x}$ & $\mathrm{x}$ & & $\mathrm{R}$ & G \\
\hline Saltator coerulescens & $\mathrm{x}$ & & & $\mathrm{x}$ & $\mathrm{R}$ & IF \\
\hline Saltator atriceps & $\mathrm{x}$ & & & $\mathrm{x}$ & $\mathrm{R}$ & IF \\
\hline Passerina caerulea & & $\mathrm{x}$ & $\mathrm{x}$ & & W & GI \\
\hline Dives dives & $\mathrm{x}$ & $\mathrm{x}$ & $\mathrm{x}$ & $\mathrm{x}$ & $\mathrm{R}$ & GIF \\
\hline Quiscalus mexicanus & & $\mathrm{x}$ & $\mathrm{x}$ & $\mathrm{x}$ & $\mathrm{R}$ & $\mathrm{O}$ \\
\hline Molothrus aeneus & & $\mathrm{x}$ & $\mathrm{x}$ & & $\mathrm{R}$ & GIF \\
\hline Icterus spurius & & $\mathrm{x}$ & $\mathrm{x}$ & & $\mathrm{T}$ & GIF \\
\hline Icterus galbula & $\mathrm{x}$ & & & & W & IF \\
\hline Psarocolius wagleri & $\mathrm{x}$ & & $\mathrm{x}$ & $\mathrm{x}$ & $\mathrm{R}$ & GF \\
\hline Psarocolius montezuma & $\mathrm{x}$ & & $\mathrm{x}$ & $\mathrm{x}$ & $\mathrm{R}$ & GF \\
\hline Euphonia hirundinacea & $\mathrm{x}$ & & & $\mathrm{x}$ & $\mathrm{R}$ & IF \\
\hline
\end{tabular}


Estatus: residente (R), visitante de invierno (W), transitorio (T), residente de verano (S).

Dieta y subdieta: insectívoras (I), frugívoras (F), nectarívoras (N), carnívoras (C), granívoras (G), omnívoras (O), carnívorainsectívora (CI), carnívoras-insectívoras-frugívoras (CIF), granívoras-frugívoras (GF), granívoras-insectívoras (GI), granívoras-insectívoras-frugívoras (GIF), insectívoras-frugívoras (IF) e insectívoras-nectarívoras (IN). 